\title{
De Garengeot's Hernia Treated With a Hybrid Approach: A Case Report
}

\author{
Mohamed Alkashty ${ }^{1}$, Ben Dickinson ${ }^{1}$, Giovanni D. Tebala ${ }^{2}$ \\ ${ }^{1}$ Department of General Surgery, Wexham Park Hospital, Frimley Health NHS Foundation Trust, Slough; ${ }^{2}$ Department of General Surgery, \\ John Radcliffe Hospital, Oxford University Hospitals NHS Foundation Trust, Oxford, United Kingdom
}

De Garengeot's hernia happens when an inflamed or ischemic appendix is located within an incarcerated femoral hernia. We hereby report a case of De Garengeot's hernia treated with a combined open and laparoscopic approach. An 80-yearold male presented to the emergency department with a 1-day history of a tender right inguinal mass. A computed tomography scan revealed a direct right inguinal hernia containing an incarcerated appendix. At surgery, the diagnosis of a strangulated appendix within a femoral hernia was made. To avoid a wide disruption of the right groin region, the ischemic appendix was reduced into the abdomen and removed laparoscopically. The femoral defect was treated by open plug repair. De Garengeot's hernia may represent a surgical challenge. A combined open and laparoscopic approach is a good option in these cases.

Keywords: Femoral hernia; Appendicitis; De Garengeot's hernia

\section{INTRODUCTION}

Femoral hernias account for only $3 \%$ of all hernias. In a mere $0.5 \%$ to $5 \%$ of these cases, the appendix travels through the femoral canal and presents as a so-called De Garengeot's hernia, first described by the eponymous French surgeon in the 18th Century [1]. Due to the narrow and rigid ring around the herniated organs when in the femoral canal, the likelihood of incarceration and strangulation is much higher than in inguinal hernias $[2,3]$. The surgical management of De Garengeot's hernias is not yet standardized. We hereby report a case of a femoral hernia containing a strangulated appendix, treated with a double inguinal and laparoscopic approach.

Received: Mar 31, 2020 - Revised: Sep 21, 2020 - Accepted: Sep 21, 2020 Correspondence to: Giovanni D. Tebala, M.D., FRCS, FACS

Emergency Surgery Unit, Department of General Surgery, John Radcliffe Hospital, Oxford University Hospitals NHS Foundation Trust, Headley Way, Headington, Oxford OX3 9DU, United Kingdom

Tel: +44-7555-349834

E-mail: giovanni.tebala@ouh.nhs.uk

ORCID: https://orcid.org/0000-0001-7152-4096

(C) 2021 The Korean Society of Coloproctology

This is an open-access article distributed under the terms of the Creative Commons Attribution NonCommercial License (https://creativecommons.org/licenses/by-nc/4.0) which permits unrestricted noncommercial use, distribution, and reproduction in any medium, provided the original work is properly cited.

\section{CASE REPORT}

An 80-year-old man presented to the emergency department with a 1-day history of a right inguinal mass that was soft and tender with no impulse on coughing. Attempts to reduce the mass were unsuccessful. Computed tomography (CT) scan (Fig. 1) revealed a direct right inguinal hernia containing an incarcerated appendix, surrounded by inflammatory changes and fluid. The caecum was pulled infero-laterally, but the intra-abdominal appearance was otherwise unremarkable. An emergency surgical operation was offered and accepted. After general anesthesia and right inguinal incision, it was evident that the non-reducible groin mass was a strangulated femoral hernia containing a partially gangrenous appendix (De Garengeot's hernia) (Fig. 2). The sac was opened but it was impossible to reach the caecum from the femoral canal, so we decided to remove the appendix by laparoscopy. At laparoscopic exploration, the femoral hernia containing the appendix was evident (Fig. 3). The appendix was retrieved into the abdomen with gentle traction and removed laparoscopically. The small peritoneal orifice leading to the femoral canal was closed with a figure-of- 8 intracorporeal vicryl stitch. Although there was no gross intraperitoneal contamination, an abundant washout was performed, and a drain was left in the pelvis. After evacuating the pneumoperitoneum and removing the trocars, an open plug repair of the femoral hernia was performed. The pa- 


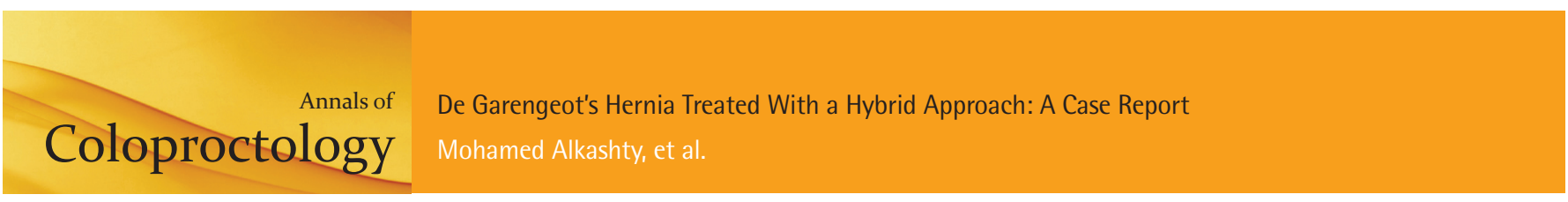

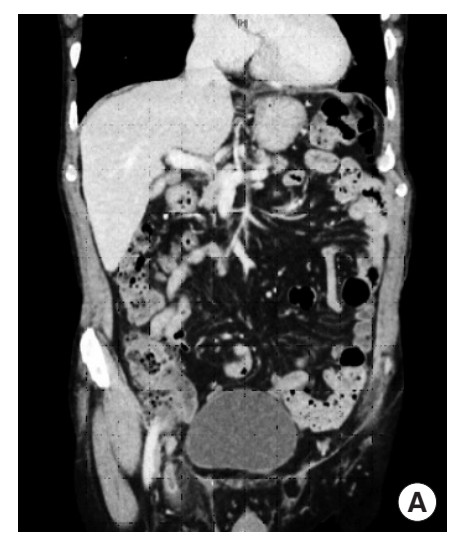

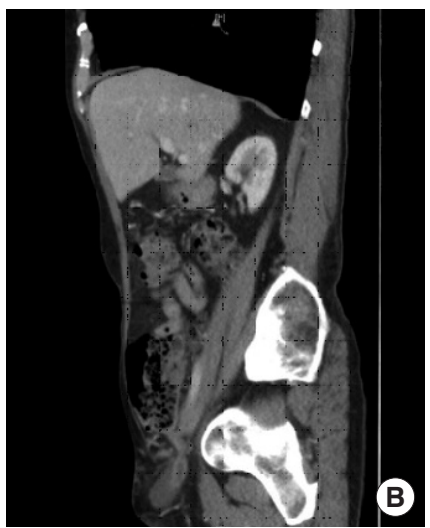

Fig. 1. (A) Coronal and (B) sagittal view of computed tomography scan of the abdomen and pelvis showing an incarcerated right inguinal hernia containing an inflamed appendix. The caecal pole was pulled infero-laterally. No signs of obstruction or perforation.

tient had a smooth recovery and was discharged on postoperative day 1 . He was well, with no infection or recurrence 3 months following surgery.

\section{DISCUSSION}

A femoral hernia containing the appendix was first described by René-Jacques Croissant de Garengeot in 1731 [1] and was given the eponymous moniker "De Garengeot hernia" by Akopian and Alexander [4] in 2005. Its true incidence is difficult to calculate as only few cases have been reported in the literature. Diagnosis of De Garengeot's hernia is challenging and it is often discovered intraoperatively. Physical signs are important in diagnosing an incarcerated or strangulated hernia but cannot specifically identify a De Garengeot's hernia [3]. Ultrasound, CT, and magnetic resonance imaging are valuable, but the rate of misdiagnosis remains high. In our case, a CT scan correctly identified the presence of an inflamed appendix within a hernia sac but misidentified it as an inguinal hernia.

Almost invariably, De Garengeot's hernias present acutely when incarcerated or strangulated, raising the possibility that the acute appendicular inflammation is in actual facts secondary to the incarceration and ischemia. Due to the narrow opening of the femoral ring, the inflammatory process is usually confined within a small compartment, thus promoting local abscess formation and decreasing the risk of generalized peritonitis [4]. In our case, the patient was not overtly septic and his inflammatory markers were normal; however, other authors have reported the presence of sepsis in around 50\% of patients [5].

A review of the recent literature confirms that the choice of surgical technique cannot be standardized and is usually based on the anatomical and clinical findings as well as the local availability of laparoscopic expertise. In the absence of gross peritoneal in-

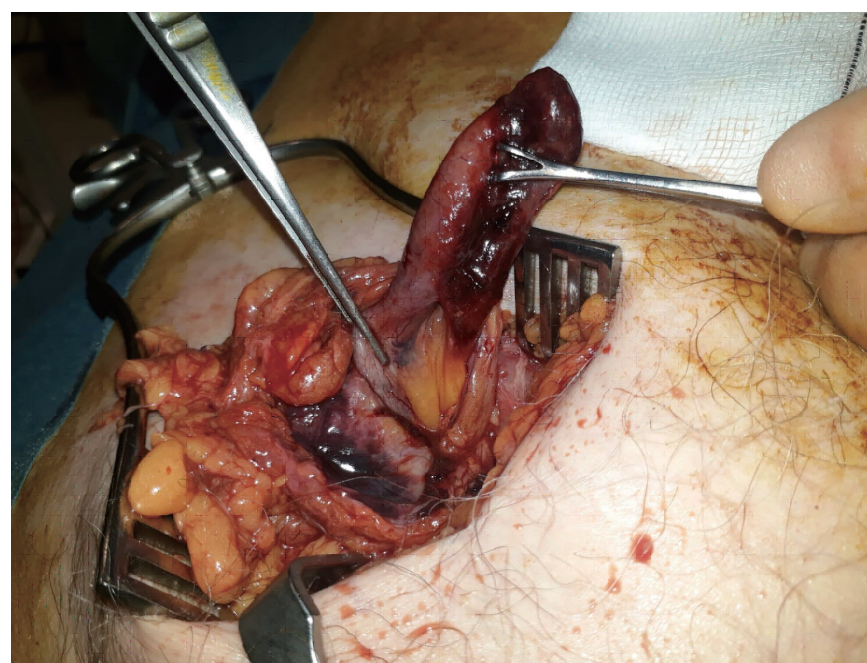

Fig. 2. Intraoperative findings. Groin incision. Gangrenous appendix in the femoral hernia sac.

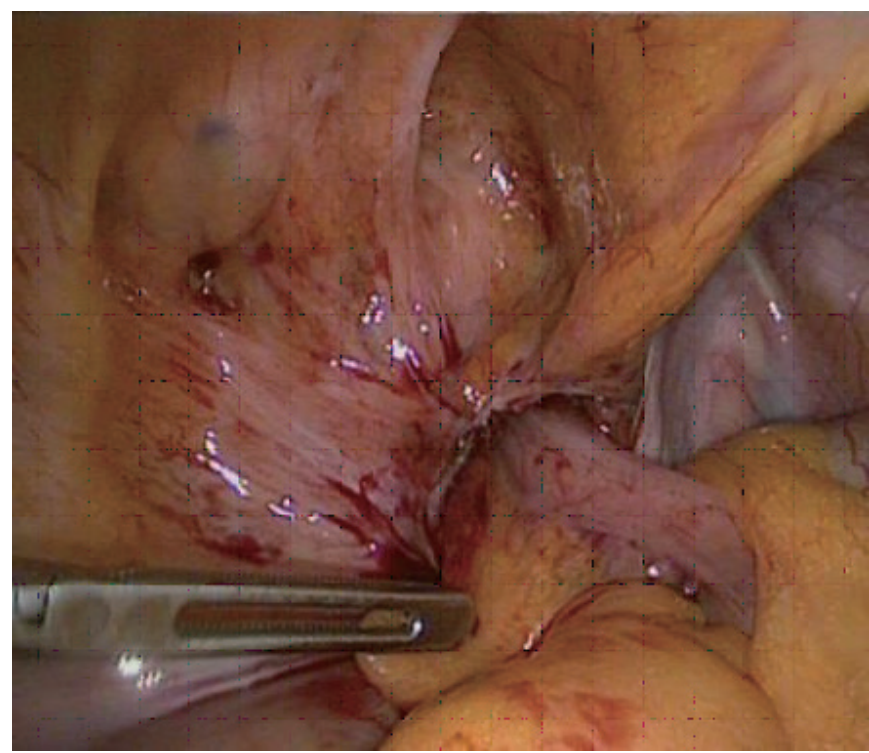

Fig. 3. Intraoperative findings. Laparoscopic exploration. Strangulated femoral hernia containing the appendix.

flammation, it may be possible to remove the appendix and repair the hernia from a groin incision, but difficulties can arise if the appendicular basis cannot be reached from the groin. In our case, although the appendix was quite mobile, we were unable to reach its basis, prompting the decision to remove the appendix by laparoscopy. This permitted a thorough examination of the abdomen and pelvis, without the need for a formal laparotomy. As the femoral canal was already opened, we opted to stitch the peritoneal defect by laparoscopy and repair the femoral hernia by open surgery, as an eventual laparoscopic repair would have added further anatomical dissection and the risk of mesh infection. On the con- 
trary, we aimed at separating the 2 compartments as soon as possible, to avoid cross-contamination.

We feel that laparoscopy can represent a valid procedural option and should be considered as the gold standard as it associates the benefits of a good surgical exposure with those of a minimally invasive approach. In patients with abdominal symptoms only, the operation may be performed entirely by laparoscopy, without the need for a groin exploration. In our case, however, the principal complaint was of a tender inguinal mass, so our initial choice of access was inguinal. We maintain that, in cases of purely laparoscopic approach, there should be a debate as to whether the femoral hernia should be concurrently repaired alongside the appendectomy using a transabdominal preperitoneal technique. Although there is no strong evidence to support or deny this choice, we believe that in the presence of intraperitoneal inflammation, as in acute appendicitis or ischemic gangrene, it is preferable to keep the abdominal and crural/inguinal compartments separated to avoid cross-contamination. Occasionally, a primary mesh repair of the femoral hernia may not be indicated at all, and a 2-stage operation may be preferable, with appendectomy (open or laparoscopic) associated with crural debridement and drain, followed by mesh repair of the femoral hernia at a later stage.

Although rare, De Garengeot's hernia can represent a significant surgical challenge. The paucity of cases precludes a standardized operative procedure and various methods can be used. Laparoscopy plays an important diagnostic and therapeutic role. Special consideration must be paid to the presence of crural and/or peritoneal contamination as it may change the operative strategy.

\section{REFERENCES}

1. De Garengeot RJ. Traite des operations de chirurgie. 2nd ed. Paris: Chez Huarte; 1731.

2. Kalles V, Mekras A, Mekras D, Papapanagiotou I, Al-Harethee W, Sotiropoulos G, et al. De Garengeot's hernia: a comprehensive review. Hernia 2013;17:177-82.

3. Barbaros U, Asoglu O, Seven R, Kalayci M. Appendicitis in incarcerated femoral hernia. Hernia 2004;8:281-2.

4. Akopian G, Alexander M. De Garengeot hernia: appendicitis within a femoral hernia. Am Surg 2005;71:526-7.

5. Linder S, Linder G, Månsson C. Treatment of de Garengeot's hernia: a meta-analysis. Hernia 2019;23:131-41. 